\title{
Chromosomal phenotypes and submicroscopic abnormalities
}

\author{
Koen Devriendt and Joris R. Vermeesch* \\ Center for Human Genetics, University Hospital Leuven, Herestraat 49, B-3000 Leuven, Belgium \\ *Correspondence to: E-mail: joris.vermeesch@uz.kuleuven.ac.be
}

Date received (in revised form): 17th October 2003

\begin{abstract}
The finding, during the last decade, that several common, clinically delineated syndromes are caused by submicroscopic deletions or, more rarely, by duplications, has provided a powerful tool in the annotation of the human genome. Since most microdeletion/microduplication syndromes are defined by a common deleted/duplicated region, abnormal dosage of genes located within these regions can explain the phenotypic similarities among individuals with a specific syndrome. As such, they provide a unique resource towards the genetic dissection of complex phenotypes such as congenital heart defects, mental and growth retardation and abnormal behaviour. In addition, the study of phenotypic differences in individuals with the same microdeletion syndrome may also become a treasury for the identification of modifying factors for complex phenotypes. The molecular analysis of these chromosomal anomalies has led to a growing understanding of their mechanisms of origin. Novel tools to uncover additional submicroscopic chromosomal anomalies at a higher resolution and higher speed, as well as the novel tools at hand for deciphering the modifying factors and epistatic interactors, are 'on the doorstep' and will, besides their obvious diagnostic role, play a pivotal role in the genetic dissection of complex phenotypes.
\end{abstract}

Keywords: microdeletion, low copy repeat, syndrome, microarray

\section{Clinical features of microdeletion syndromes}

The clinical features of the microdeletion syndromes are highly diverse and complex (Table 1). Microduplications appear to be rarer, and will not be discussed in detail here. For some syndromes, patients present with a major organ malformation, such as a congenital heart defect. Characteristic heart defects are seen for each microdeletion syndrome, eg supravalvular aortic stenosis (SVAS) and peripheral pulmonary stenosis (PPS) in the Williams syndrome (7q11 deletion), conotruncal heart malformations (such as tetralogy of Fallot, truncus arteriosus and interrupted aortic arch) in the 22q11.2 deletion (causing the DiGeorge/velocardiofacial [VCF] syndrome) and atrioventricular septal defect or pulmonary stenosis with atrial septal defect in the 8p23.1 deletion. Characteristic facial features are frequently present and may aid in the diagnosis. As with most chromosomal aberrations, retarded physical growth is also commonly observed - with the notable exception of Sotos syndrome - where somatic overgrowth is a key feature. Besides the physical findings, many individuals with a microdeletion come to medical attention because of developmental delay. The delay ranges from borderline (eg in VCF syndrome) to severe (as in the Angelman syndrome; AS). It is of interest that these learning difficulties often have a characteristic profile, most typically seen in the Williams syndrome, where individuals have a large discrepancy between their verbal and performance intelligence. ${ }^{1}$ Moreover, several microdeletions cause a characteristic behavioural phenotype. ${ }^{2}$ The most studied example is the Williams syndrome, where individuals are described as 'overfriendly-though-anxious' and lack social judgement skills. ${ }^{1}$ In the Smith-Magenis syndrome (SMS; del17p11.2) and in the chromosome 8p23.1 deletion, severe sleeping disturbances and self-injury are common. ${ }^{3,4}$ The Prader-Willi syndrome (PWS; del15q11-13), gives rise to an insatiable appetite, leading to morbid obesity, with frequent temper tantrums. ${ }^{2}$ It is also recognised that individuals with either VCF syndrome or PWS are at increased risk for psychiatric disease, specifically psychoses. ${ }^{5,6}$

Given the wide range of phenotypic manifestations, clinical recognition of the microdeletion syndromes has become part of general medical practice, beyond just paediatrics and clinical genetics.

\section{Cause of microdeletions}

Following the discovery of microdeletion syndromes, it was noted that in the majority of affected individuals, the deletion for each specific syndrome has a uniform size. This suggested 
Table I. Characteristics of common microdeletion syndromes

\begin{tabular}{|c|c|c|c|c|c|}
\hline Syndrome & $\begin{array}{l}\text { Chromosome } \\
\text { location }\end{array}$ & Deletion incidence & Parental origin & $\begin{array}{l}\text { Deletion size } \\
(\mathrm{Mb})\end{array}$ & Gene \\
\hline Sotos & $5 q 35$ & ND & Paternal (90\%) & 2.2 & $\begin{array}{l}\text { NSDI } \\
(10 \%)\end{array}$ \\
\hline Williams & $7 q 11.23$ & $1 / 20,000-1 / 50,000$ & Equal & 1.6 & CGS \\
\hline $8 p$ deletion & $8 p 23.1$ & ND & Maternal & 5 & CGS \\
\hline Prader-Willi & $15 q \mid 1.2-13$ & $1 / 20,000$ & Paternal & 3.5 & CGS \\
\hline Angelman & $15 q 1.2-13$ & $1 / 20,000$ & Maternal & 3.5 & $\begin{array}{l}\text { UBE3A } \\
\qquad(10-15 \%)\end{array}$ \\
\hline Smith-Magenis & $|7 p| \mid .2$ & $\mathrm{I} / 25,000$ & Equal & 4 & RAII (ND) \\
\hline Neurofibromatosis I & $|7 q| 1.2$ & $1 / 40,000-1 / 80,000$ & Maternal & 1.5 & $\begin{array}{l}\text { NFI } \\
\qquad(90-95 \%)\end{array}$ \\
\hline Velocardiofacial & $22 q 11.2$ & $1 / 4,000$ & Equal & $3(1.5)$ & CGS \\
\hline
\end{tabular}

Abbreviations: ND, not determined; CGS, contiguous gene deletion syndrome

an underlying chromosomal structural feature in the region that confers a predisposition to the loss of small chromosomal fragments. Subsequently, it was proven that region-specific low-copy repeats (LCRs) flank all the microdeletions mentioned in Table 1. The deletion breakpoints cluster within these flanking LCRs. It is now well established that non-allelic homologous recombination by both inter- and intrachromosomal events cause the deletions. ${ }^{7-10}$ The interchromosomal rearrangements arise by an unequal crossing-over during meiosis I through paralogous LCRs between homologous chromosomes (Figure 1). Intrachromosomal rearrangements may occur by several mechanisms, including LCR mispairingmediated unequal sister chromatid exchange and the formation of an intrachromosomal loop that is also mediated by LCRs within a single chromatid. ${ }^{11,12}$ Intrachromosomal rearrangements can occur either during meiosis or postzygotically. Since mosaics for microdeletion syndromes have only rarely been detected, however, it is most likely that the majority of the intrachromosomal rearrangements occur during the meiotic period.

The reciprocal product of the deletion caused by homologous recombination by misaligned flanking LCRs is a duplication. The frequency of duplications should equal the frequency of microdeletions. This paradigm is exemplified by two syndromes, Charcot-Marie-Tooth disease type 1A (CMT1A) and hereditary neuropathy with liability to pressure palsies (HNPP), with an incidence of 1/3,000 and 1/ 7,000, respectively. While CMT1A is caused by a microduplication of $17 \mathrm{p} 11.2$, HNPP is caused by a microdeletion in the same region. Surprisingly, however, reciprocal microdeletion/microduplication syndromes like CMT1A/HNPP are rare, or even never observed, for the other microdeletion syndromes. Duplications have now been described for the $22 \mathrm{q} 11$ region, ${ }^{13}$ the $15 \mathrm{q} 11-13$ region $^{14}$ and the $17 \mathrm{p} 11.2-12$ region. ${ }^{15}$ It is intriguing that only a few duplications have been detected so far. One possibility is that duplications would be lethal. In general, however, chromosome duplications cause a less severe phenotype than deletions (in unbalanced translocations, the phenotype is usually determined more by the deleted chromosome fragment than by the duplication). Alternatively, it may be that there is no distinct phenotype, and that individuals with duplications do not come to medical attention. If this were so, however, one would expect that the duplications would remain present in the general population and that they would be detected during the many tests and screenings that are currently performed. Another possibility is that carriers of duplications may be less reproductively fit, so the duplication may not embed in the population. In conclusion, at present, there is no satisfactory explanation for the apparently low frequency of microduplication syndromes.

While it is now well established that LCRs in the genome induce a susceptibility for the generation of microdeletions, it remains unclear why some rearrangements occur more often than others. It has been estimated that 5-10 per cent of the genome is composed of LCRs; ${ }^{16-18}$ however, only a fraction of these seem to cause recurrent rearrangements. For Sotos syndrome, a thus far unexplained difference exists in the occurrence of a microdeletion in chromosome $5 \mathrm{q} 35$ between Japanese and non-Japanese individuals. While at least 50 per cent of Japanese patients carry a 5 q35 microdeletion, ${ }^{19,20}$ these microdeletions appear to be exceptional in non-Japanese patients. ${ }^{21}$ Determining the factors that influence a predisposition for rearrangements is currently the topic of active 
(A) INTERCHROMOSOMAL RECOMBINATION

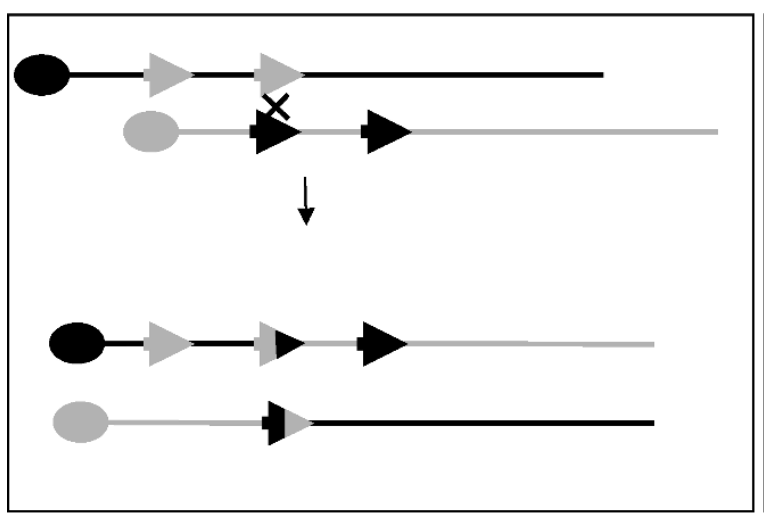

(B) INTRACHROMOSOMAL RECOMBINATION
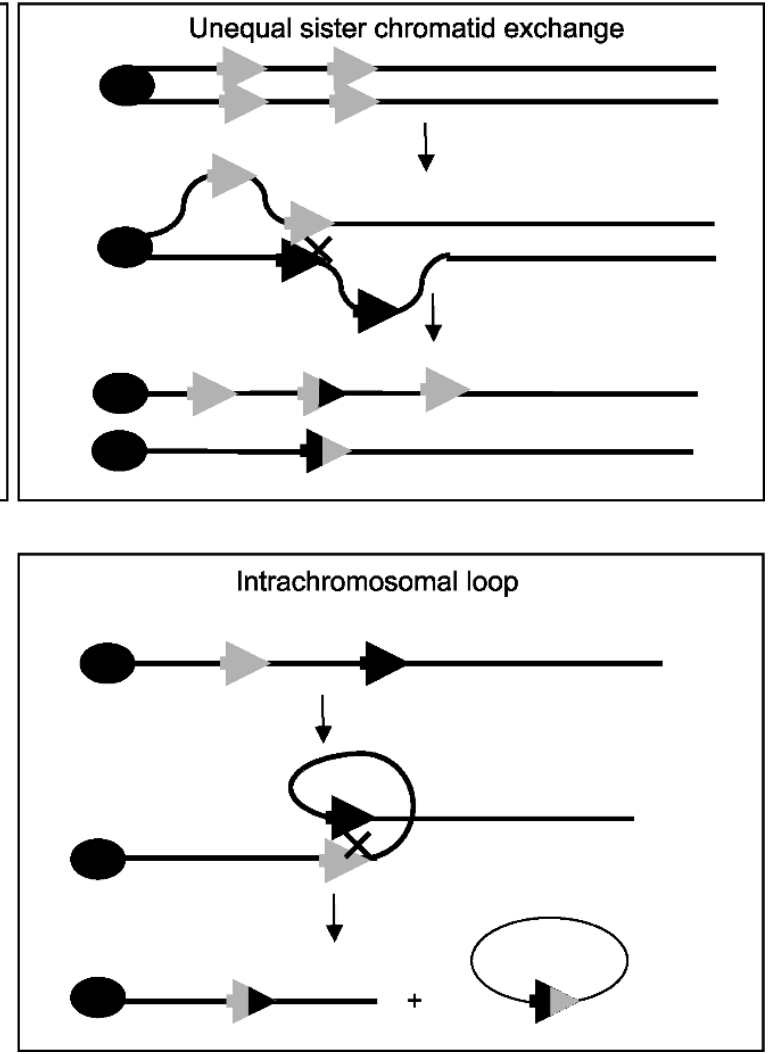

Figure I. Schematic representation of nonallelic homologous recombination by both inter- and intrachromosomal events. The black and grey lines represent the homologous chromosomes. The arrows represent low copy repeats (LCRs). The different tints of the LCRs are used to indicate the position of the cross-over. In (A) they represent the LCR on the two different chromosomes; in (B) they represent either the LCR on the two different sister-chromatids or the two different LCR on a single sister chromatid. The $X$ indicates the site of the cross-over.

research. The major elements known to influence the frequency of such rearrangements will be discussed below.

\section{The size of the LCRs}

In general, LCRs flanking the commonly deleted regions range between 200 and $500 \mathrm{~kb}$ in size. Apart from these flanking LCRs, however, smaller paralogous sequences exist within the commonly deleted regions of several syndromes. Such sequences exist within DiGeorge/VCF syndrome (22q11.2), ${ }^{22}$ SMS (del17p11.2), ${ }^{23}$ PWS/AS (del15q11-13) ${ }^{24}$ and neurofibromatosis type 1 (NF1) (del17q11.2) ${ }^{25}$ commonly deleted regions. These sequences have been shown to predispose to microdeletions as well, albeit at a lower frequency. Hence, the size of the paralogous sequences appears, not surprisingly, to influence the frequency by which meiotic misalignment and subsequent uneven cross-overs do occur.

\section{The sequence identity among the LCRs}

It seems likely that sequence identity will influence the frequency of nonallelic homologous recombination (NAHR).
Evolutionary studies and sequence analysis of the LCRs has unveiled some intriguing features. The LCRs seem to have appeared during primate speciation..$^{22,23,26-29}$ Why such large homology segments are preserved in the human genome and why different LCRs seem to populate different chromosomes is not obvious. It is also unclear how the homology is preserved. Sequence analysis in the CMT1A/HNPP flanking LCRs has shown the occurrence of gene conversion flanking the recombination hotspots. ${ }^{8,30}$ It is possible that gene conversion homogenises LCRs and influences the rate of NAHR. This hypothesis deserves closer scrutiny.

\section{The inherent capacity of the LCR sequence to initiate homologous recombination}

The parental origin of microdeletions and/or duplications has been investigated in several genomic disorders. Deletions in Williams syndrome, VCF syndrome and SMS showed equal paternal and maternal origins. ${ }^{7,9,31,32}$ By contrast, deletions in CMT1A disease and Sotos syndrome occur more often in the 
paternally derived chromosomes than in the maternally derived chromosomes. ${ }^{8,33-35}$ Microdeletions in the NF1 locus and the $8 \mathrm{p}$ region are predominantly of maternal origin. ${ }^{10,36,37}$ These observations are most easily explained by differences in the capacity of certain sequences to initiate homologous recombination. Recombination rates vary greatly across the genome, from zero to $8.8 \mathrm{cM} / \mathrm{Mb}$. Moreover, recombination rates and sites vary between males and females, ${ }^{38}$ and sexspecific recombination hotspots have been mapped. ${ }^{39,40}$ In fact, the NF1-flanking LCRs and 8p olfactory receptor gene clusters flanking the $8 \mathrm{p}$ terminal microdeletion appear to be female-specific recombination sites. Lopez-Correa et al. found that 46 per cent of the NF1 microdeletion breakpoints cluster in a $2 \mathrm{~kb}$ region within the flanking LCRs, supporting the thesis of the presence of a recombination hotspot. ${ }^{41}$ Similarly, Reiter et al. discovered a $2 \mathrm{~kb}$ region where the breakpoints cluster has been found in 75 per cent of CMT1A patients and 84 per cent of HNPP patients. ${ }^{42}$ The CMT1A and Sotos syndrome flanking sequences may thus represent male-specific recombination hotspots.

\section{The orientation of the sequence between the LCRs}

Parental submicroscopic inversion polymorphisms between the LCRs flanking the microdeletion regions have recently been demonstrated to be present in several microdeletion syndromes. An inversion polymorphism on chromosome $8 \mathrm{p}$ was present in all mothers whose offspring had a deletion of the corresponding olfactory receptor gene cluster. ${ }^{37,43,44}$ This polymorphism turned out to be present in the heterozygous state in 26 per cent of a population of European descent. A comparable inversion polymorphism was present in one-third of parents of origin of probands with the $1.5 \mathrm{Mb}$ deletion at 7q11.23 causing Williams-Beuren syndrome. ${ }^{45}$ At the Emery Dreifuss muscular dystrophy region, a similar inversion polymorphism has been detected in 33 per cent of females. ${ }^{46}$ Gimelli et al. showed that four out of six mothers of AS patients having the BP2/3 deletion carry an inversion. ${ }^{24}$ This inversion appears to be present at 9 per cent in the general population. These observations raise the question of whether parental inversion polymorphisms are a common phenomenon in all microdeletion syndromes. A recent report suggests that, at least for the most common deletion syndrome, the 22q11 microdeletion, inversion polymorphisms do not exist or are very rare. ${ }^{47}$ Another question raised is whether the presence of an inversion increases the risk of producing microdeleted offspring. Although the answer is likely to be positive, the risk level is probably only marginally increased. Considering that about one-quarter of the population carries the $8 \mathrm{p}$ inversion, the occurrence of $\operatorname{del}(8 \mathrm{p})$ is extremely rare; however, this risk may be variable for inversion polymorphisms at different loci, and further studies are needed to address this question.

\section{Genetic dissection of the common features in microdeletion syndromes}

The phenotype of a number of microdeletion syndromes is by and large determined by haploinsufficiency of a single gene within the deleted region. In NF1, the majority of patients carry a mutation in a single gene (the NF1 gene), and a microdeletion is found in only about $5-10$ per cent of cases. The phenotype of deletion patients is more severe, with more pronounced facial dysmorphism and more pronounced developmental delay. ${ }^{25,48}$ Interestingly, these individuals also have a higher number of neurofibromata at a younger age and a higher risk of malignant peripheral nerve sheet tumours, ${ }^{48,49}$ indicating the presence of one or more genes in this region contributing to this. In AS, patients with a mutation in the UBE3A gene are clinically almost indistinguishable from those with a microdeletion. ${ }^{50}$ Nevertheless, as a group, deletion patients are more severely affected, with a higher incidence of seizures, microcephaly and more severe developmental delay. This suggests that other genes in the deleted region contribute to the phenotype, and that the GABA receptor genes in 15q11-13 are possible candidate genes for the more pronounced epilepsy. ${ }^{51}$ In Sotos syndrome, most features can be explained by haploinsufficiency for the NSD1 gene, whereas in some individuals, additional manifestations are probably related to other genes in the deleted region. ${ }^{19-21}$

In other instances, different phenotypic manifestations are related to haploinsufficiency for different, contiguous genes within the deleted region. The prototype is Williams syndrome. The heart defects seen with these syndromes (typically PPS and SVAS) are caused by haploinsufficiency of the elastin gene, since mutations in this gene have been found in families with autosomal dominant PPS and/or SVAS. ${ }^{52}$ These individuals lack any of the other features of Williams syndrome, however, indicating that these must be caused by the deletion of other contiguous genes. At present, no other gene has been linked to the other features of this syndrome, such as hypercalcaemia, developmental delay or the characteristic behavioural phenotype. Dissection of the genetic components of contiguous gene syndromes (CGSs) has proven challenging for microdeletion syndromes, given the almost uniform size of the deletions in the majority of patients. For most microdeletion syndromes, however, rare cases exist with smaller or overlapping deletions and with partial or complete phenotypic manifestations. For example, in SMS (del17p11.2), genotype-phenotype correlations in rare individuals carrying a smaller or overlapping deletion allowed a $1.1 \mathrm{Mb}$ critical deletion region to be defined for the phenotype. ${ }^{53}$ This led to the identification of mutations in the RAI1 gene in three individuals with the SMS phenotype but without a deletion. ${ }^{54}$ Likewise, a few individuals have been identified carrying deletions in the chromosome 7q11 region which are smaller than 
or overlap the common Williams deletion region, and which manifest only some of the Williams syndrome features. ${ }^{55,56}$

In addition to explaining the phenotypic manifestations in patients carrying a microdeletion syndrome, the identified genes are also excellent candidate susceptibility genes for the more common multifactorial phenotypes in men, such as organ malformations, intelligence and behaviour. For example, individuals with a del22q11 have an increased risk of developing psychosis. Two candidate genes for psychoses are located within the deleted region on chromosome 22q11.2, the catechol-O-methyltransferase (COMT) gene and the proline dehydrogenase (PRODH) gene. The COMT gene plays a role in dopamine neurotransmitter degradation, and abnormal function of dopaminergic pathways is thought to play a role in psychosis. ${ }^{57}$ Thus far, no convincing studies exist that show the association of the common functional val-met polymorphism and the risk for psychosis. ${ }^{57}$ More recently, however, Schifman et al. have shown the association of a COMT haplotype (but not the val-met polymorphism) with schizophrenia in the general population. ${ }^{58}$ Mutations in the PRODH gene have been detected in some patients with schizophrenia; ${ }^{59}$ moreover, polymorphisms near the PRODH gene were associated with schizophrenia in three independent study samples. ${ }^{60}$

\section{Genetic dissection of the differences in microdeletion syndromes}

Despite the deletions having a uniform size in the majority of patients with the same microdeletion syndrome, clinical expression can vary widely. The best example to illustrate this with is the phenotype caused by del22q11, which has been described as a number of different syndromes, such as the DiGeorge syndrome (hypoparathyroidism, thymus hypoplasia, conotruncal heart defect and facial dysmorphism), the VCF syndrome (cleft palate, conotruncal heart defect, learning difficulties and characteristic facial features) and, in the Japanese literature, the conotruncal anomaly facies syndrome (CTAFS).

Since haploinsufficiency of the deleted gene(s) alone cannot explain clinical variability in most cases, other modifying factors must exist. As for all complex genetic features or disorders, phenotype is influenced by additional genetic and environmental factors. In a microdeletion syndrome, the phenotype is determined by a single gene with major effect and influenced by a limited number of additional genetic and environmental factors with a small effect. This is a less complex situation than the more common multifactorial features, where the phenotype is determined by multiple environmental and genetic factors, each with a small effect. Because of this, and given their frequent occurrence, the microdeletion syndromes present a unique opportunity to dissect modifying factors of complex phenotypes.
The most obvious candidate modifiers are mutations/ polymorphisms in the non-deleted allele of genes within the deleted region (Figure 2). The most extreme presentation of this is a recessive disorder, where a microdeletion unmasks a mutation in the non-deleted allele. For example, BernardSoulier syndrome (BSS) can be caused by homozygous mutations in the GP1Bb gene, located in chromosome 22q11. This disorder features platelet dysfunction, with elevated platelet volumes and lowered counts. In one patient with a microdeletion in 22q11 and BSS, a mutation was detected in the non-deleted GP1Bb allele. ${ }^{61}$ Another example is a patient with AS and recessive oculocutaneous albinism type 2 (OCA2), caused by absence of the $P$ gene. Fridman et al. found this gene to be located in the deleted region on the maternal chromosome 15q11.2-13 in AS, and a deletion within the $P$ gene to be present on the paternal chromosome. ${ }^{62}$

In contrast to mutations, polymorphisms are an almost general feature of most human genes. Therefore, polymorphisms in the non-deleted allele are excellent candidates for genetic modifiers. As stated above, individuals with del22q11 have an increased risk of developing psychosis, an observation which has been seen in approximately 10 per cent of cases. Polymorphisms in the COMT and PRODH genes are obvious candidates for association studies in VCF syndrome individuals with and without psychosis.

Besides mutations or polymorphisms in the non-deleted allele, the phenotype can also be influenced by polymorphisms in genes located elsewhere in the genome. In VCF syndrome, between 50 and 75 per cent of cases have a congenital heart defect, despite the presence of a uniform-sized deletion. Mice lacking the 164 isoform of the vascular endothelial growth factor (vegf) proteins display a phenotype almost identical to the tbx1 knockout mouse. ${ }^{63}$ In addition, in zebrafish, an interaction between tbx1 and vegf could be demonstrated in knockdown experiments. ${ }^{63}$ These data suggested that human VEGF might be a modifier for the heart defects in individuals with del22q11. This was confirmed by showing that functional polymorphisms in the VEGF gene promotor/5'UTR on chromosome $6 \mathrm{p} 12$ are associated with decreased VEGF expression and confer an increased risk for the development of a congenital heart defect in individuals with del22q11. ${ }^{63}$ This represents the first known genetic modifier outside the deleted region for a malformation seen in a microdeletion syndrome.

In addition to genetic modifiers, phenotype is also influenced by environmental factors. For example, individuals with PWS develop morbid obesity unless put on a very restrictive diet from very early on. ${ }^{64}$ Most features and diseases have a multifactorial origin and, in both the VCF syndrome and PWS, intelligence is correlated with parental intelligence quotients, indicating a multifactorial influence on intelligence in these microdeletion syndromes. ${ }^{65,66}$ Interestingly, monozygotic twins with del22q11 but discordant for a heart defect have repeatedly been reported. ${ }^{67}$ Possible explanations include post-zygotic mutations, epigenetic changes and stochastic factors. 
METHOD

EXAMPLES

A. Genetic dissection of common features

a.

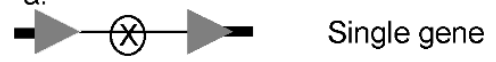

$\stackrel{b .}{*} \otimes x-$ cGs

B. Molecular understanding of modifying factors

a. Autosomal recessive

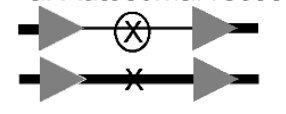

Mutation in

non-deleted allele

b. Haplotype non-deleted allele

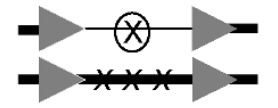

Polymorphisms in non-deleted alleles
Overlapping/smaller deletions
\& candidate gene
mutation analysis

Mutation analysis

of candidate genes

Association studies in patients with deletion: non-deleted alleles

Association studies in patients with deletion:

- candidate genes

- whole genome linkage

- disequilibrium screening
Angelman (UBE3A)

Sotos (NSD1)

Neurofibromatosis (NF1)

Williams (Elastin, ...)

\section{c. Non-allelic genetic modifiers}

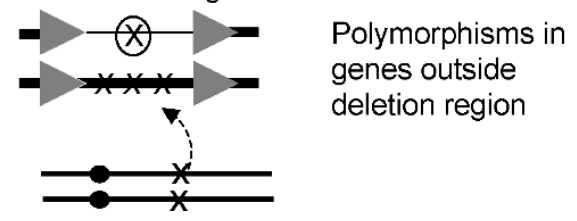

Figure 2. Strategies towards the dissection the genetic components of microdeletion syndromes. Abbreviations: CGS, contiguous gene syndrome; COMT, catechol-O-methyltransferase; PRODH, proline dehydrogenase; VEGF, vascular endothelial growth factor.

\section{Towards whole genome microdeletion/microduplication screens}

As discussed above, the study of microdeletions is a powerful tool in the annotation of the human genome. The discovery of these microdeletion syndromes has so far occurred haphazardly. It is likely that some of the so far unexplained clinically recognisable syndromes with a 'chromosomal' phenotype can be explained by a recurrent microdeletion or microduplication. Moreover, a significant proportion of individuals with unexplained developmental disorders will probably be explained by random microdeletions and/or duplications. Evidence for this hypothesis comes from the many sporadic reports of submicroscopic interstitial chromosomal rearrangements. The introduction of a subtelomeric screen enabled a molecular diagnosis to be made for about 5 per cent of the idiopathic mentally retarded population. ${ }^{68}$ These observations raise the expectation that a genome-wide microdeletion screen may well pick up various unknown intrachromosomal aberrations. Techniques that will enable genome-wide aneuploidy analysis are thus needed and are being developed.
The most advanced of these techniques is matrix or array comparative genomic hybridisation (CGH). ${ }^{69-71}$ This technique combines the advantages of the resolution power of fluorescent in situ hybridisation (FISH) with the screening capacity of the chromosome scans used by classical cytogenetic techniques. In this technique, genomic DNA from patient and control are differentially labelled with two fluorescent dyes. The labelled DNAs are co-hybridised to DNA arrays, which consist of DNA spots derived from clones containing genomic DNA fragments - usually bacteria artificial chromosomes (BACs). Chromosomal deletions are detected by fluorescent intensity ratios of the spots containing less patient dye compared with control dye, and vice versa for duplications. Chromosomal imbalances across the genome can thus be quantified and their position determined. The resolution of array CGH can be controlled and is dependent on a combination of the number, size and map positions of the DNA elements within the array. ${ }^{71-73}$ Array CGH has been successfully applied to analyse a variety of constitutional aberrations. Just recently, the technique has been introduced into the clinical genetics laboratory. ${ }^{73-76}$

It seems likely that within the next five years, aneuploidy genome scanning tools will become integrated into routine 
diagnostic cytogenetic laboratories. Certainly, this will have a major impact on clinical genetics. In addition, correlations of defined phenotypic manifestations with the deletion or duplication of specific genes will provide a unique opportunity to further the annotation of the human genome.

\section{Acknowledgments}

Koen Devriendt is a Senior Clinical Investigator in the Fund for Scientific Research-Flanders (FWO, Vlaanderen). He is supported by grants from the University of Leuven (OT) and FWO, Vlaanderen.

\section{References}

1. Donnai, D. and Karmiloff-Smith, A. (2000), 'Williams syndrome: From genotype through to the cognitive phenotype', Am. J. Med. Genet. Vol. 97, pp. $164-171$

2. Cassidy, S.B. and Morris, C.A. (2002), 'Behavioral phenotypes in genetic syndromes: Genetic clues to human behaviour', Adv. Pediatr. Vol. 49, pp. 59-86.

3. Smith, A.C., Dykens, E. and Greenberg, F. (1998), 'Behavioral phenotype of Smith-Magenis syndrome (del 17p11.2)', Am. J. Med. Genet. Vol. 81, pp. 179-185.

4. Claeys, I., Holvoet, M., Eyskens, B. et al. (1997), 'A recognisable behavioural phenotype associated with terminal deletions of the short arm of chromosome 8', Am. J. Med. Genet. Vol. 74, pp. 515-520.

5. Clarke, D.J. (1993), 'Prader-Willi syndrome and psychoses', Br. J. Psychiatry Vol. 163 , pp. $680-684$.

6. Murphy, K.C. and Owen, M.J. (2001), 'Velo-cardio-facial syndrome: A model for understanding the genetics and pathogenesis of schizophrenia', Br. J. Psychiatry Vol. 179, pp. 397-402.

7. Urban, Z., Helms, C., Fekete, G. et al. (1996), '7q11.23 deletions in Williams syndrome arise as a consequence of unequal meiotic crossover', Am. J. Hum. Genet. Vol. 59, pp. 958-962.

8. Lopes, J., Vandenberghe, A., Tardieu, S. et al. (1997), 'Sex-dependent rearrangements resulting in CMT1A and HNPP', Nat. Genet. Vol. 17, pp. 136-137.

9. Baumer, A., Dutly, F., Balmer, D. et al. (1998), 'High level of unequal meiotic crossovers at the origin of the 22q11. 2 and 7q11.23 deletions', Hum. Mol. Genet. Vol. 7, pp. 887-894.

10. Lopez, C.C., Brems, H., Lazaro, C. et al. (2000), 'Unequal meiotic crossover: A frequent cause of NF1 microdeletions', Am. J. Hum. Genet. Vol. 66, pp. 1969-1974.

11. Ji, Y., Eichler, E.E., Schwartz, S. et al. (2000), 'Structure of chromosomal duplicons and their role in mediating human genomic disorders', Genome Res. Vol. 10, pp. 597-610

12. Stankiewicz, P., Shaw, C.J., Dapper, J.D. et al. (2003), 'Genome architecture catalyzes nonrecurrent chromosomal rearrangements', Am. J. Hum. Genet. Vol. 72, pp. 1101-1116.

13. Ensenauer, R.E., Adeyinka, A., Flynn, H.C. et al. (2003), 'Microduplication 22q11.2, an emerging syndrome: Clinical, cytogenetic, and molecular analysis of thirteen patients', Am. J. Hum. Genet. Vol. 73, pp. $1027-1040$.

14. Roberts, S.E., Dennis, N.R., Browne, C.E. et al. (2002), 'Characterisation of interstitial duplications and triplications of chromosome 15q11-q13', Hum. Genet. Vol. 110, pp. 227-234.

15. Potocki, L., Chen, K.S., Park, S.S. et al. (2000), 'Molecular mechanism for duplication $17 \mathrm{p} 11.2$ - the homologous recombination reciprocal of the Smith-Magenis microdeletion', Nat. Genet. Vol. 24, pp. 84-87.

16. Bailey, J.A., Yavor, A.M., Massa, H.F. et al. (2001), 'Segmental duplications: Organization and impact within the current human genome project assembly', Genome Res. Vol. 11, pp. 1005-1017.
17. Bailey, J.A., Gu, Z., Clark, R.A. et al. (2002), 'Recent segmental duplications in the human genome', Science Vol. 297, pp. 1003-1007.

18. Lupski, J.R. (2003), 2002 Curt Stern Award Address. 'Genomic disorders recombination-based disease resulting from genomic architecture', $\mathrm{Am}$. J. Hum. Genet. Vol. 72, pp. 246-252.

19. Kurotaki, N., Imaizumi, K., Harada, N. et al. (2002), 'Haploinsufficiency of NSD1 causes Sotos syndrome', Nat. Genet. Vol. 30, pp. 365-366.

20. Nagai, T., Matsumoto, N., Kurotaki, N. et al. (2003), 'Sotos syndrome and haploinsufficiency of NSD1: Clinical features of intragenic mutations and submicroscopic deletions', J. Med. Genet. Vol. 40, pp. 285-289.

21. Douglas, J., Hanks, S., Temple, I.K. et al. (2003), 'NSD1 mutations are the major cause of Sotos syndrome and occur in some cases of Weaver syndrome but are rare in other overgrowth phenotypes', Am. J. Hum. Genet. Vol. 72, pp. 132-143.

22. Shaikh, T.H., Kurahashi, H. and Emanuel, B.S. (2001), 'Evolutionarily conserved low copy repeats (LCRs) in 22q11 mediate deletions, duplications, translocations and genomic instability: An update and literature review', Genet. Med. Vol. 3, pp. 6-13.

23. Park, S.S., Stankiewicz, P., Bi, W. et al. (2002), 'Structure and evolution of the Smith-Magenis syndrome repeat gene clusters, SMS-REPs', Genome Res. Vol. 12, pp. 729-738.

24. Gimelli, G., Pujana, M.A., Patricelli, M.G. et al. (2003), 'Genomic inversions of human chromosome 15q11-q13 in mothers of Angelman syndrome patients with class II (BP2/3) deletions', Hum. Mol. Genet. Vol. 12, pp. 849-858.

25. Lopez, C.C., Brems, H., Lazaro, C. et al. (1999), 'Molecular studies in 20 submicroscopic neurofibromatosis type 1 gene deletions', Hum. Mutat. Vol. 14, pp. 387-393.

26. Kiyosawa, H. and Chance, P.F. (1996), 'Primate origin of the CMT1AREP repeat and analysis of a putative transposon-associated recombinational hotspot', Hum. Mol. Genet. Vol. 5, pp. 745-753.

27. Christian, S.L., Fantes, J.A., Mewborn, S.K. et al. (1999), 'Large genomic duplicons map to sites of instability in the Prader-Willi/Angelman syndrome chromosome region (15q11-q13)', Hum. Mol. Genet. Vol. 8, pp. $1025-1037$.

28. Valero, M.C., de Luis, O., Cruces, J. et al. (2000), 'Fine-scale comparative mapping of the human $7 \mathrm{q} 11.23$ region and the orthologous region on mouse chromosome 5G: The low-copy repeats that flank the WilliamsBeuren syndrome deletion arose at breakpoint sites of an evolutionary inversion(s)', Genomics Vol. 69, pp. 1-13

29. Inoue, K., Dewar, K., Katsanis, N. et al. (2001), 'The 1.4 Mb CMT1A duplication/HNPP deletion genomic region reveals unique genome architectural features and provides insights into the recent evolution of new genes', Genome Res. Vol. 11, pp. 1018-1033.

30. Reiter, L.T., Hastings, P.J., Nelis, E. et al. (1998), 'Human meiotic recombination products revealed by sequencing a hotspot for homologous strand exchange in multiple HNPP deletion patients', Am. J. Hum. Genet. Vol. 62, pp. 1023-1033.

31. Dutly, F. and Schinzel, A. (1996), 'Unequal interchromosomal rearrangements may result in elastin gene deletions causing the WilliamsBeuren syndrome', Hum. Mol. Genet. Vol. 5, pp. 1893-1898.

32. Shaw, C.J., Bi, W. and Lupski, J.R. (2002), 'Genetic proof of unequal meiotic crossovers in reciprocal deletion and duplication of 17p11.2', Am. J. Hum. Genet. Vol. 71, pp. 1072-1081.

33. Palau, F., Lofgren, A., De Jonghe, P. et al. (1993), 'Origin of the de novo duplication in Charcot-Marie-Tooth disease type 1A: Unequal nonsister chromatid exchange during spermatogenesis', Hum. Mol. Genet. Vol. 2, pp. 2031-2035.

34. Bort, S., Martinez, F. and Palau, F. (1997), 'Prevalence and parental origin of de novo $1.5 \mathrm{Mb}$ duplication in Charcot-Marie-Tooth disease type 1A', Am. J. Hum. Genet. Vol. 60, pp. 230-233.

35. Miyake, N., Kurotaki, N. and Sugawara, H. et al. (2003), 'Preferential paternal origin of microdeletions caused by prezygotic chromosome or chromatid rearrangements in Sotos syndrome', Am. J. Hum. Genet. Vol. 72, pp. 1331-1337.

36. Lazaro, C., Gaona, A., Ainsworth, P. et al. (1996), 'Sex differences in mutational rate and mutational mechanism in the NF1 gene in neurofibromatosis type 1 patients', Hum. Genet. Vol. 98, pp. 696-699. 
37. Giglio, S., Broman, K.W., Matsumoto, N. et al. (2001), 'Olfactory receptor-gene clusters, genomic-inversion polymorphisms and common chromosome rearrangements', Am. J. Hum. Genet. Vol. 68, pp. 874-883.

38. Yu, A., Zhao, C., Fan, Y. et al. (2001), 'Comparison of human genetic and sequence-based physical maps', Nature Vol. 409, pp. 951-953.

39. Robinson, W.P. and Lalande, M. (1995), 'Sex-specific meiotic recombination in the Prader-Willi/Angelman syndrome imprinted region', Hum. Mol. Genet. Vol. 4, pp. 801-806.

40. Badge, R.M., Yardley, J., Jeffreys, A.J. et al. (2000), 'Crossover breakpoint mapping identifies a subtelomeric hotspot for male meiotic recombination', Hum. Mol. Genet. Vol. 9, pp. 1239-1244.

41. Lopez-Correa, C., Dorschner, M., Brems, H. et al. (2001), 'Recombination hotspot in NF1 microdeletion patients', Hum. Mol. Genet. Vol. 10, pp. 1387-1392.

42. Reiter, L.T., Murakami, T., Koeuth, T. et al. (1996), 'A recombination hotspot responsible for two inherited peripheral neuropathies is located near a mariner transposon-like element', Nat. Genet. Vol. 12 , pp. $288-297$.

43. Giglio, S., Calvari, V., Gregato, G. et al. (2002), 'Heterozygous submicroscopic inversions involving olfactory receptor-gene clusters mediate the recurrent t(4;8)(p16;p23) translocation', Am. J. Hum. Genet. Vol. 71, pp. $276-285$.

44. Vermeesch, J.R., Thoelen, R. and Salden, I. et al. (2003), 'Mosaicism $\operatorname{del}(8 \mathrm{p}) /$ inv $\operatorname{dup}(8 \mathrm{p})$ in a dysmorphic female infant: A mosaic formed by a meiotic error at the $8 \mathrm{p}$ OR gene and an independent terminal deletion event', J. Med. Genet. Vol. 40(8), e93.

45. Osborne, L.R., Li, M., Pober, B. et al. (2001), 'A 1.5 million-base pair inversion polymorphism in families with Williams-Beuren syndrome', Nat. Genet. Vol. 29, pp. 321-325.

46. Small, K., Iber, J. and Warren, S.T. (1997), 'Emerin deletion reveals a common X-chromosome inversion mediated by inverted repeats', Nat. Genet. Vol. 16, pp. 96-99.

47. Gebhardt, G.S., Devriendt, K., Thoelen, R. et al. (2003), 'No evidence for a parental inversion polymorphism predisposing to rearrangements at 22q11.2 in the DiGeorge/velocardiofacial syndrome', Eur. J. Hum. Genet. Vol. 11, pp. 109-111.

48. Kayes, L.M., Burke, W., Riccardi, V.M. et al. (1994), 'Deletions spanning the neurofibromatosis 1 gene: Identification and phenotype of five patients', Am. J. Hum. Genet. Vol. 54, pp. 424-436.

49. De Raedt, T., Brems, H., Wolkenstein, P. et al. (2003), 'Elevated risk for MPNST in NF1 microdeletion patients', Am. J. Hum. Genet. Vol. 72, pp. $1288-1292$.

50. Clayton-Smith, J. and Laan, L. (2003), 'Angelman syndrome: A review of the clinical and genetic aspects', J. Med. Genet. Vol. 40, pp. 87-95.

51. Minassian, B.A., DeLorey, T.M., Olsen, R.W. et al. (1998), 'Angelman syndrome: Correlations between epilepsy phenotypes and genotypes', Ann. Neurol. Vol. 43, pp. 485-493.

52. Tassabehji, M., Metcalfe, K., Donnai, D. et al. (1997), 'Elastin: genomic structure and point mutations in patients with supravalvular aortic stenosis', Hum. Mol. Genet. Vol. 6, pp. 1029-1036.

53. Bi, W., Yan, J., Stankiewicz, P. et al. (2002), 'Genes in a refined Smith-Magenis syndrome critical deletion interval on chromosome $17 \mathrm{p} 11.2$ and the syntenic region of the mouse', Genome Res. Vol. 12, pp. $713-728$.

54. Slager, R.E., Newton, T.L., Vlangos, C.N. et al. (2003), 'Mutations in RAI1 associated with Smith-Magenis syndrome', Nat. Genet. Vol. 33, pp. 466-468.

55. Frangiskakis, J.M., Ewart, A.K., Morris, C.A. et al. (1996), 'LIM-kinase1 hemizygosity implicated in impaired visuospatial constructive cognition', Cell Vol. 86, pp. 59-69.

56. Tassabehji, M., Metcalfe, K., Karmiloff-Smith, A. et al. (1999), 'Williams syndrome: Use of chromosomal microdeletions as a tool to dissect cognitive and physical phenotypes', Am. J. Hum. Genet. Vol. 64, pp. $118-125$.
57. Murphy, K.C., Jones, L.A. and Owen, M.J. (1999), 'High rates of schizophrenia in adults with velo-cardio-facial syndrome', Arch. Gen. Psychiatry Vol. 56, pp. 940-945.

58. Shifman, S., Bronstein, M., Sternfeld, M. et al. (2002), 'A highly significant association between a COMT haplotype and schizophrenia', Am. J. Hum. Genet. Vol. 71, pp. 1296-1302.

59. Jacquet, H., Raux, G., Thibaut, F. et al. (2002), 'PRODH mutations and hyperprolinemia in a subset of schizophrenic patients', Hum. Mol. Genet. Vol. 11, pp. 2243-2249.

60. Liu, H., Heath, S.C., Sobin, C. et al. (2002), 'Genetic variation at the 22q11 PRODH2/DGCR6 locus presents an unusual pattern and increases susceptibility to schizophrenia', Proc. Natl. Acad. Sci. USA Vol. 99, pp. 3717-3722.

61. Ludlow, L.B., Schick, B.P., Budarf, M.L. et al. (1996), 'Identification of a mutation in a GATA binding site of the platelet glycoprotein Ibbeta promoter resulting in the Bernard-Soulier syndrome', J. Biol. Chem. Vol. 271, pp. 22076-22080.

62. Fridman, C., Hosomi, N., Varela, M.C. et al. (2003), 'Angelman syndrome associated with oculocutaneous albinism due to an intragenic deletion of the $P$ gene', Am. J. Med. Genet. Vol. 119A, pp. 180-183.

63. Stalmans, I., Lambrechts, D., De Smet, F. et al. (2003), 'VEGF: A modifier of the del22q11 (DiGeorge) syndrome?', Nat. Med. Vol. 9, pp. 173-182.

64. Descheemaeker, M.J., Swillen, A., Plissart, L. et al. (1994), 'The PraderWilli syndrome: A self-supporting program for children, youngsters and adults', Genet. Couns. Vol. 5, pp. 199-205.

65. Swillen, A., Devriendt, K., Legius, E. et al. (1997), 'Intelligence and psychosocial adjustment in velocardiofacial syndrome: a study of 37 children and adolescents with VCFS', J. Med. Genet. Vol. 34, pp. 453-458.

66. Malich, S., Largo, R.H., Schinzel, A. et al. (2000), 'Phenotypic heterogeneity of growth and psychometric intelligence in Prader-Willi syndrome: Variable expression of a contiguous gene syndrome or parentchild resemblance?', Am. J. Med. Genet. Vol. 91, pp. 298-304.

67. Vincent, M.C., Heitz, F., Tricoire, J. et al. (1999), '22q11 deletion in DGS/VCFS monozygotic twins with discordant phenotypes', Genet. Couns. Vol. 10, pp. 43-49.

68. Knight, S.J., Regan, R., Nicod, A. et al. (1999), 'Subtle chromosomal rearrangements in children with unexplained mental retardation', Lancet Vol. 354, pp. 1676-1681.

69. Bentz, M., Plesch, A., Stilgenbauer, S. et al. (1998), 'Minimal sizes of deletions detected by comparative genomic hybridization', Genes Chromosomes Cancer Vol. 21, pp. 172-175.

70. Kirchhoff, M., Rose, H. and Lundsteen, C. (2001), 'High resolution comparative genomic hybridisation in clinical cytogenetics', J. Med. Genet. Vol. 38, pp. 740-744.

71. Solinas-Toldo, S., Lampel, S., Stilgenbauer, S. et al. (1997), 'Matrix-based comparative genomic hybridization: Biochips to screen for genomic imbalances', Genes Chromosomes Cancer Vol. 20, pp. 399-407.

72. Pinkel, D., Segraves, R., Sudar, D. et al. (1998), 'High resolution analysis of DNA copy number variation using comparative genomic hybridization to microarrays', Nat. Genet. Vol. 20, pp. 207-211.

73. Fiegler, H., Carr, P., Douglas, E.J. et al. (2003), 'DNA microarrays for comparative genomic hybridization based on DOP-PCR amplification of BAC and PAC clones', Genes Chromosomes Cancer Vol. 36, pp. 361-374.

74. Veltman, J.A., Jonkers, Y., Nuijten, I. et al. (2003), 'Definition of a critical region on chromosome 18 for congenital aural atresia by array CGH', $\mathrm{Am}$. J. Hum. Genet. Vol. 72, pp. 1578-1584.

75. Bruder, C.E., Hirvela, C., Tapia-Paez, I. et al. (2001), 'High resolution deletion analysis of constitutional DNA from neurofibromatosis type 2 (NF2) patients using microarray-CGH', Hum. Mol. Genet. Vol. 10, pp. 271-282.

76. Veltman, J.A., Schoenmakers, E.F., Eussen, B.H. et al. 'High-throughput analysis of subtelomeric chromosome rearrangements by use of arraybased comparative genomic hybridization', Am. J. Hum. Genet. Vol. 70, pp. $1269-1276$. 